Kragujevac Journal of Mathematics

Volume 41(2) (2017), Pages 203-217.

\title{
2-TOROIDS AND THEIR 3-TRIANGULATION
}

\author{
MILICA STOJANOVIĆ ${ }^{1}$
}

\begin{abstract}
It is known that we can always 3-triangulate (i.e. divide into tetrahedra) convex polyhedra but not always non-convex polyhedra. Here we discuss possibilities and properties of 3-triangulation of 2-toroids, i.e. polyhedra topologically equivalent to sphere with 2 handles, and develop the concepts of piecewise convex polyhedra and graph of connection.
\end{abstract}

\section{INTRODUCTION}

It is known that there is a possibility to divide any polygon with $n$ vertices by $n-3$ diagonals into $n-2$ triangles without gaps and overlaps. This division is called triangulation. To do the triangulation, many different practical applications are done by using computer programs. Examples of such algorithms are given by Seidel [13], Edelsbrunner [8] and Chazelle [4]. The most interesting aspect of the problem is to design algorithms so that they are as optimal as possible.

Generalization of this process to higher dimensions is also called triangulation. It consists of dividing polyhedra (polytop) into tetrahedra (simplices) with the original vertices. Within higher dimensions, new problems arise besides the fastness of algorithm. It is proved that there is no possibility to triangulate some of non-convex polyhedra [11], [12] in a three-dimensional space, and it is also proved that different triangulations of the same polyhedron may have different numbers of tetrahedra $[1,9,14]$. Considering the smallest and the largest number of tetrahedra in triangulation (the minimal and the maximal triangulation), the authors obtained values, which linearly, resp. squarely, depend on the number of vertices. Interesting triangulations are described in the papers of Edelsbrunner, Preparata, West [9] and Sleator, Tarjan, Thurston [14]. Some characteristics of triangulation in a three-dimensional space are given by

Key words and phrases. triangulation of polyhedra, toroids, piecewise convex polyhedra. 2010 Mathematics Subject Classification. Primary: 52C17. Secondary: 52B05, 05C62.

Received: April 20, 2016.

Accepted: July 15, 2016. 
Chin, Fung, Wang [6], Develin [7] and Stojanović [20-22], and in $n$-dimensional space by Lee [10]. Algorithms for investigating triangulation in three-dimensional space are given in $[23,24]$. This problem is also related to the problems of triangulation of a set of points in a three-dimensional space $[1,9]$ and the problem of rotation distance between pair of trees [14].

By the term "polyhedron" we usually mean a simple polyhedron, topologically equivalent to sphere. But there are classes of polyhedra topologically equivalent to torus or $p$-torus (sphere with $p$ handles). Torus-like polyhedra are considered e.g. in $[2,3,5,17-19]$. By the definition of Szilassi [18], torus-like polyhedra are called toroids. Generalizing that definition, we will use the term $p$-toroids ( $p \in N$ is a given natural number) for $p$-torus-like polyhedra, and term toroids as a common name for all $p$-toroids (the Szilassi's toroids would be called 1-toroids). Since toroids are not convex, it is questionable if it is possible to 3-triangulate them. The 1-toroid with the smallest number of vertices is Császár polyhedron [2,3,5,17-19]. It has 7 vertices and is known to be triangulable with 7 tetrahedra. It is obtained as an example of polyhedron without diagonals $[5,15,16]$. Some properties of 3 -triangulation for other 1-toroids are given in [22].

In this paper, 3-triangulations of 2-toroids will be considered. Some characteristic polyhedra will be described in Section 2. In Section 3 are given some necessary definitions and properties of 3-triangulation of 1-toroids, while in Section 4, we will prove the following 3 theorems:

Theorem 1.1. For each $n \geq 10$ there exists a 2-toroid which is possible to 3triangulate.

Theorem 1.2. If it is possible to 3-triangulate 2-toroid with $n \geq 10$ vertices then, the minimal number of tetrahedra necessary for triangulation is $T_{\min } \geq n+3$.

Theorem 1.3. For each $n \geq 10$ there exist 2-toroid $P$ with $n$ vertices and $T_{\min }=n+3$.

\section{Some Characteristic Examples of Polyhedra And their 3-TRIANGULATION}

2.1. It is possible to triangulate all convex polyhedra, but this is not the case with non-convex ones. The first example of a non-convex polyhedron, which is impossible to triangulate, was given by Schönhardt [12] and referred to in [11]. This polyhedron is obtained in the following way: triangulate the lateral faces of a trigonal prism $A_{1} B_{1} C_{1} A_{2} B_{2} C_{2}$ by the diagonals $A_{1} B_{2}, B_{1} C_{2}$ and $C_{1} A_{2}$ (Fig. 1 ). Then "twist" the top face $A_{2} B_{2} C_{2}$ by a small amount in the positive direction. In such a polyhedron, none of tetrahedra with vertices in the set $\left\{A_{1}, B_{1}, C_{1}, A_{2}, B_{2}, C_{2}\right\}$ is inner, so the triangulation is not possible.

2.2. Let us now consider triangulations of a bipyramid with a triangular basis $A B C$, and apices $V_{1}$ and $V_{2}$ (Fig. 2). There are two different triangulations of this kind. The first is into two tetrahedra $V_{1} A B C$ and $V_{2} A B C$, and the second is into three: 

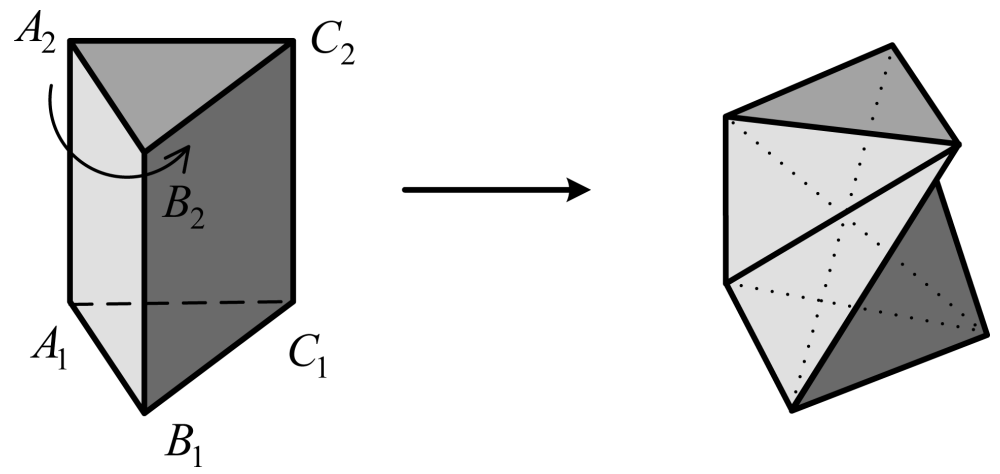

FigURE 1. Schönhardt polyhedron.

$V_{1} V_{2} A B, V_{1} V_{2} B C$ and $V_{1} V_{2} C A$. So, it is obvious that some 3 -triangulable polyhedra is possible to triangulate with different numbers of tetrahedra. That is the reason to introduce terms of minimal and maximal triangulation of a given polyhedron.
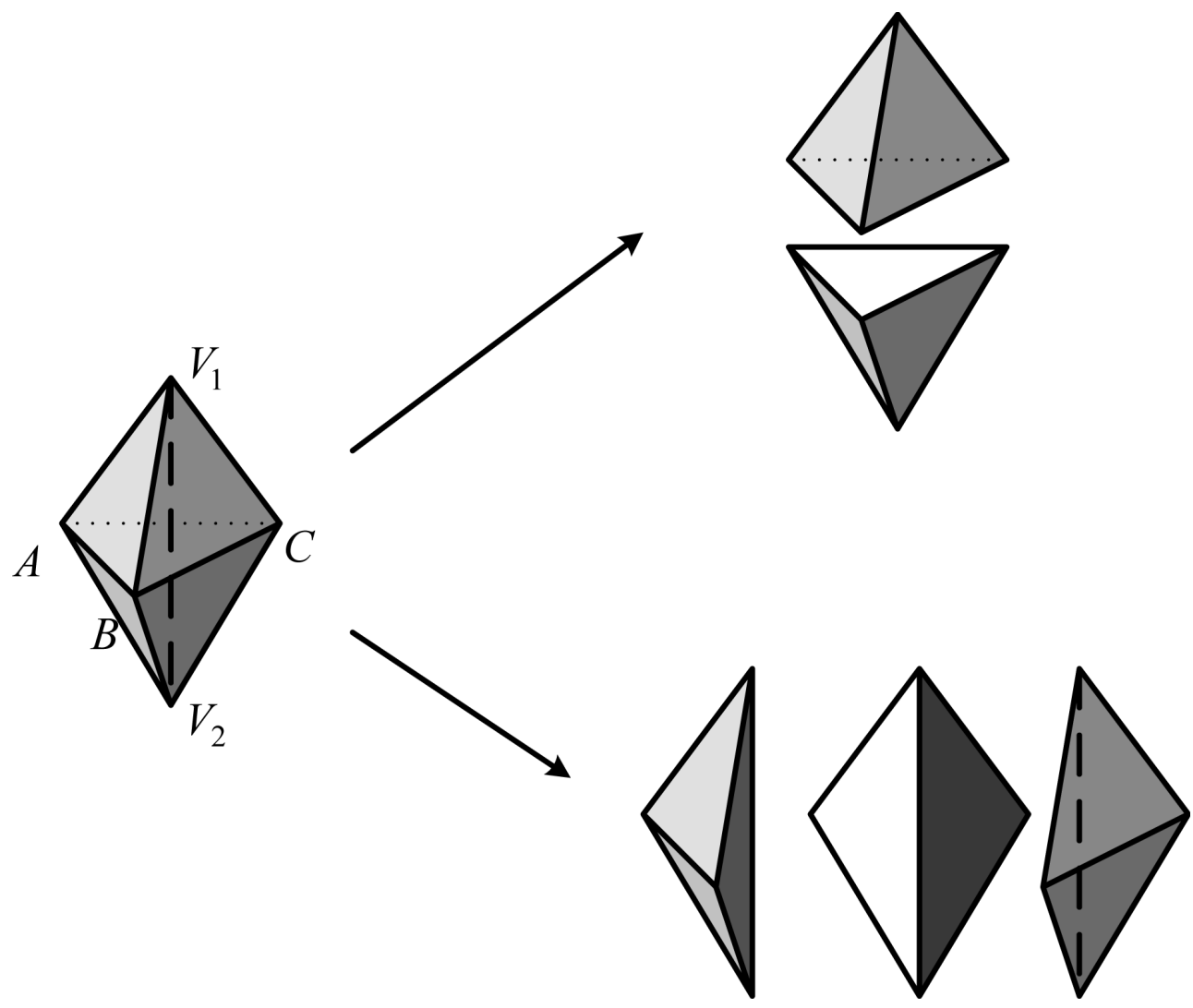

FiguRE 2. Triangulations of a trigonal bipyramid.

2.3. It is proved that the smallest possible number of tetrahedra in the triangulation of a polyhedron with $n$ vertices is $n-3$. But, it is not possible to triangulate each 
polyhedron into $n-3$ tetrahedra; for example, all triangulations of an octahedron (6 vertices) give 4 tetrahedra. Here, we will mention some examples of polyhedra triangulable with $n-3$ tetrahedra.

The pyramids with $n-1$ vertices in the basis (i.e., a total of $n$ vertices) are triangulable by doing any 2 -triangulation of the basis into $(n-1)-2=n-3$ triangles. Each of these triangles makes with the apex one of tetrahedra in 3-triangulation. If the basis of a "pyramid" is a space polygon, then it is possible to triangulate it in a similar way without taking care about convexity. For example, if we 2-triangulate lateral sides of trigonal prism $A_{1} B_{1} C_{1} A_{2} B_{2} C_{2}$ by the diagonals $B_{1} A_{2}, C_{1} A_{2}$ and $C_{1} B_{2}$ (Fig. 3) then, it is obvious that 3 -triangulation is possible with 3 tetrahedra: $A_{1} B_{1} C_{1} A_{2}, B_{1} C_{1} A_{2} B_{2}$ and $A_{2} B_{2} C_{1} C_{2}$. Here, we may assume that the basis of the trigonal pyramid is space pentagon $A_{1} B_{1} B_{2} C_{2} C_{1}$.
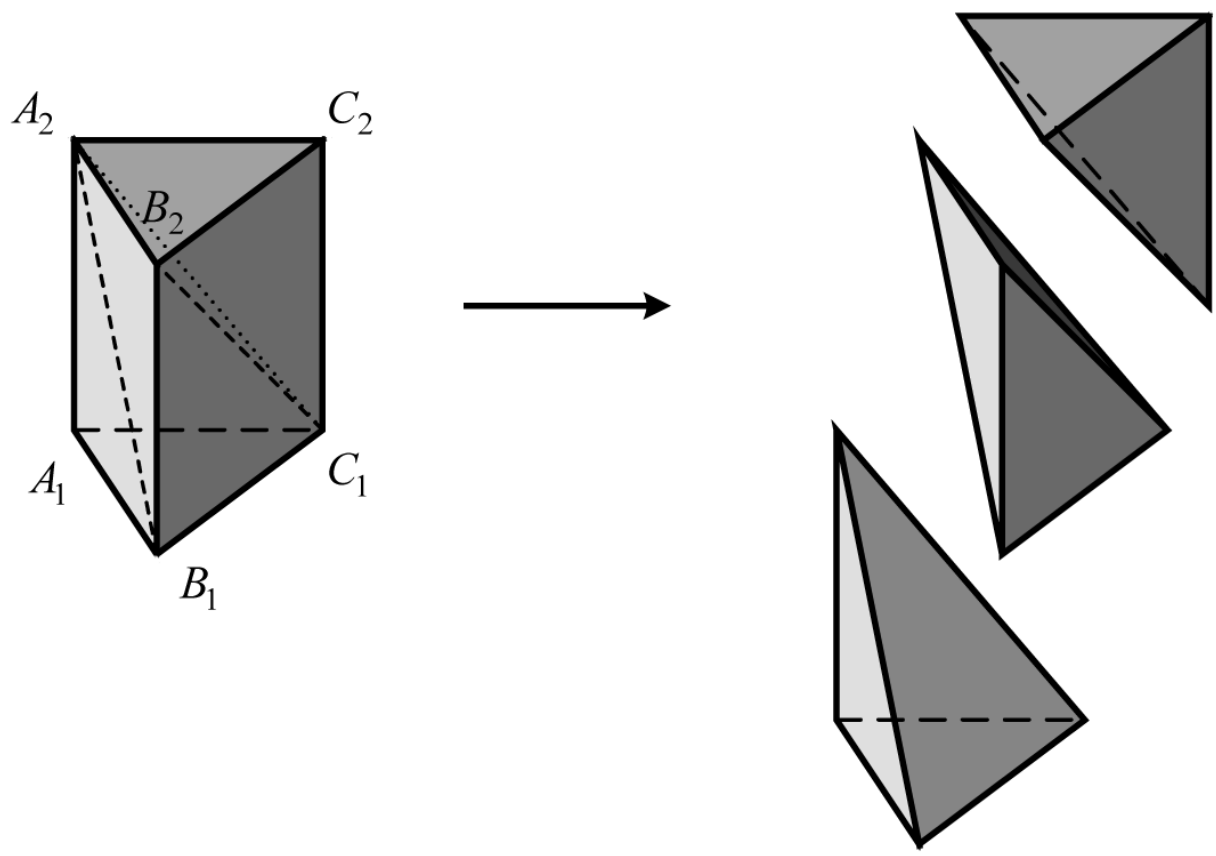

FiguRE 3. Triangulation of a trigonal prism with 3 tetrahedra.

2.4. Let us return to the two methods of triangulating a bipyramid, but this time with $n-2$ vertices in the basis (which can also be a space polygon). If we divide it into two pyramids and triangulate each of them with taking care of a common 2-triangulation of the basis, then we will obtain $2(n-4)$ tetrahedra. In the second method, each of $n-2$ tetrahedra has a common edge joining the apices of the bipyramid, and moreover, each of them contains a pair of the neighbour vertices of the basis (i.e., one of the edges of the basis). For $n=5$ (a bipyramid with a triangle basis), it 
has been found that the first method is "better", i.e., it gives a smaller number of tetrahedra. For $n=6$ (the octahedron), both methods give 4 tetrahedra, and for $n \geq 7$, the second method is "better". In figure Fig. 4 triangulations of a bipyramid with a pentagonal basis (i.e. $n=7$ ) are given. Dividing bipyramid into two pyramids leads to triangulation with 6 tetrahedra, and dividing it around the axis $V_{1} V_{2}$ gives triangulation with 5 tetrahedra.

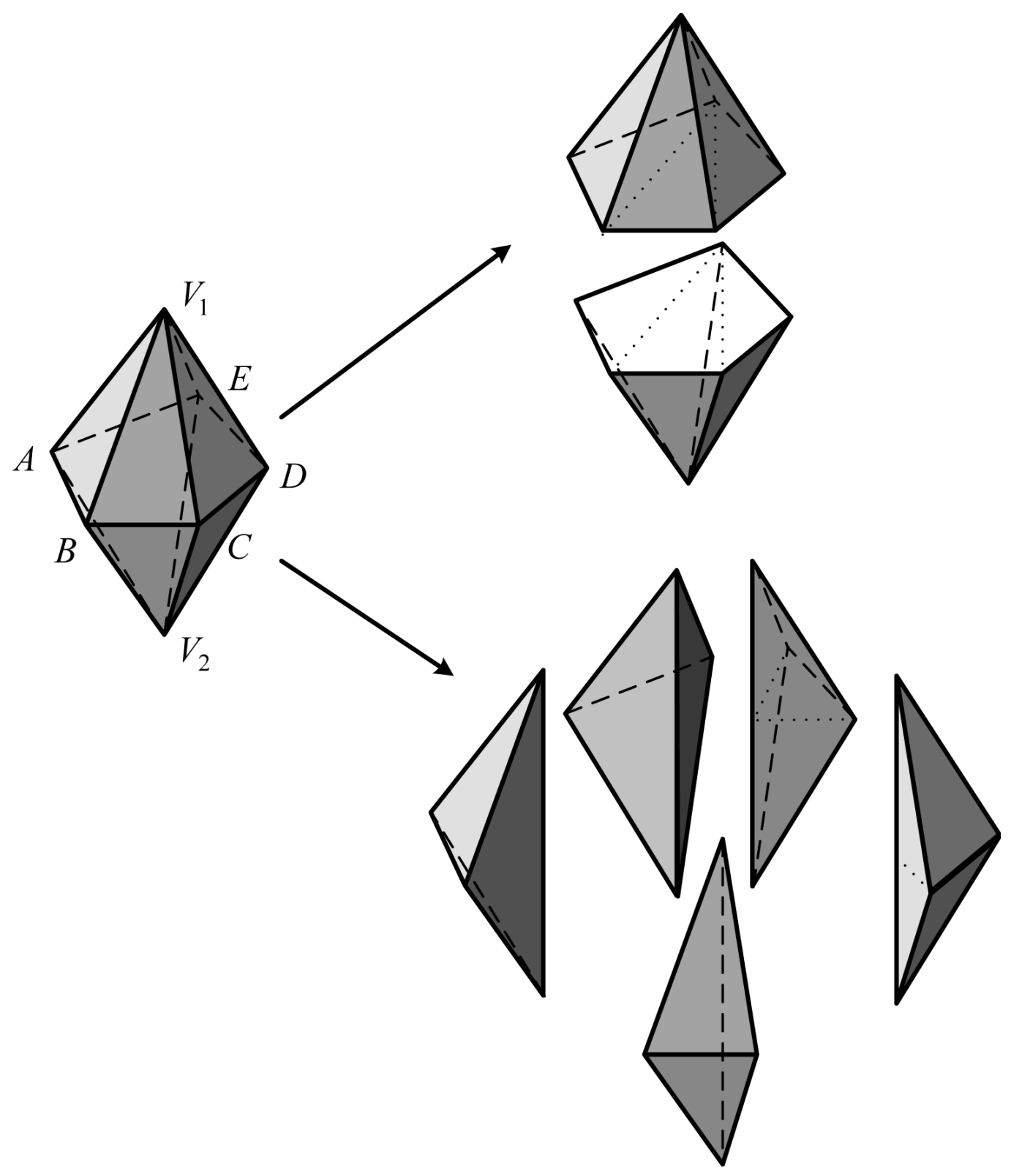

FigURE 4. Triangulations of a pentagonal bipyramid. 
2.5. In [18] Szilassi introduced term toroid. Here we will used term 1-toroid instead of toroid.

Definition 2.1. (Szilassi) An ordinary polyhedron is called 1-toroid if it is topologically torus-like (i.e. it can be converted to a torus by continuous deformation) and its faces are simply polygons.

A 1-toroid with the smallest number of vertices is the Császár polyhedron (Fig. 5). It has 7 vertices and no diagonals, i.e. each vertex is connected to the other six by edges. In [2] Bokowski and Eggert proved that Császár polyhedron has four essentially different versions. It is to be noted that in topological terms the various versions of Császár polyhedron are isomorphic - there is only one way to draw the full graph with seven vertices on the torus. Császár polyhedron is possible to 3-triangulate with 7 tetrahedra, as it is shown by Szilassi from Wolfram Demonstrations Project [19].

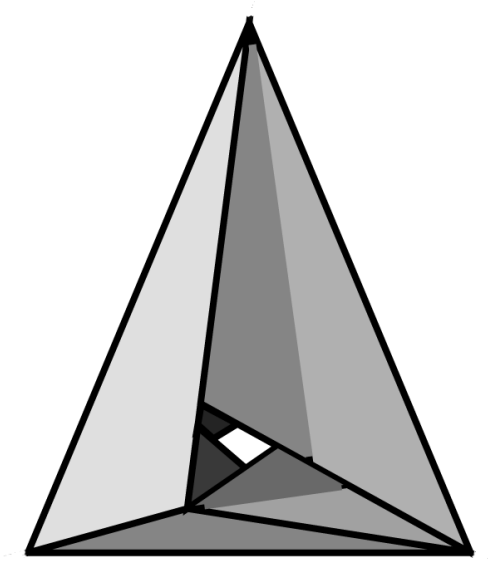

Figure 5. Császár polyhedron.

\section{Preliminaries}

In accordance with definition 2.1 there will be introduced terms 2-toroid and $p$-toroid $(p \in N)$.

\section{Definition 3.1.}

- An ordinary polyhedron is called 2-toroid if it is topologically equivalent to sphere with 2 handles (double-torus, 2-torus) and its faces are simply polygons.

- An ordinary polyhedron is called $p$-toroid, $p \in N$ is a given number, if it is topologically equivalent to sphere with $p$ handles ( $p$-torus) and its faces are simply polygons.

Let us use term toroid as a common name for all $p$-toroids. In our consideration of 3 -triangulability of toroids, we will also use the following definitions. 
Definition 3.2. Polyhedron is piecewise convex if it is possible to divide it into convex polyhedra $P_{i}, i=1, \ldots, m$, with disjunct interiors. A pair of polyhedra $P_{i}, P_{j}$ is said to be neighbour if they have common face called contact face. If polyhedra $P_{i}$ and $P_{j}$ are not neighbours, they may have a common edge $e$ or a common vertex $v$ only if there is a sequence of neighbours polyhedra $P_{i}, P_{i+1}, \ldots, P_{i+k} \equiv P_{j}$ such that the edge $e$, or the vertex $v$ belongs to each contact face $f_{l}$ common to $P_{l}$ and $P_{l+1}$, $l \in\{i, \ldots, i+k-1\}$. Otherwise, polyhedra $P_{i}$ and $P_{j}$ have not common points.

One example of piecewise convex polyhedron is given in Fig. 6. The figure shows a 1 -toroid with $n=19$ vertices, whose pieces are of two types $A$ and $B$, shown in Fig. 7. Polyhedron of type $A$ is topologically triangular prisms, while polyhedron of type $B$ is built by "gluing" a tetrahedron onto the polyhedron $A$. These two types of polyhedra with 6 , resp. 7 , vertices will be called "elemental polyhedra" in following constructions.

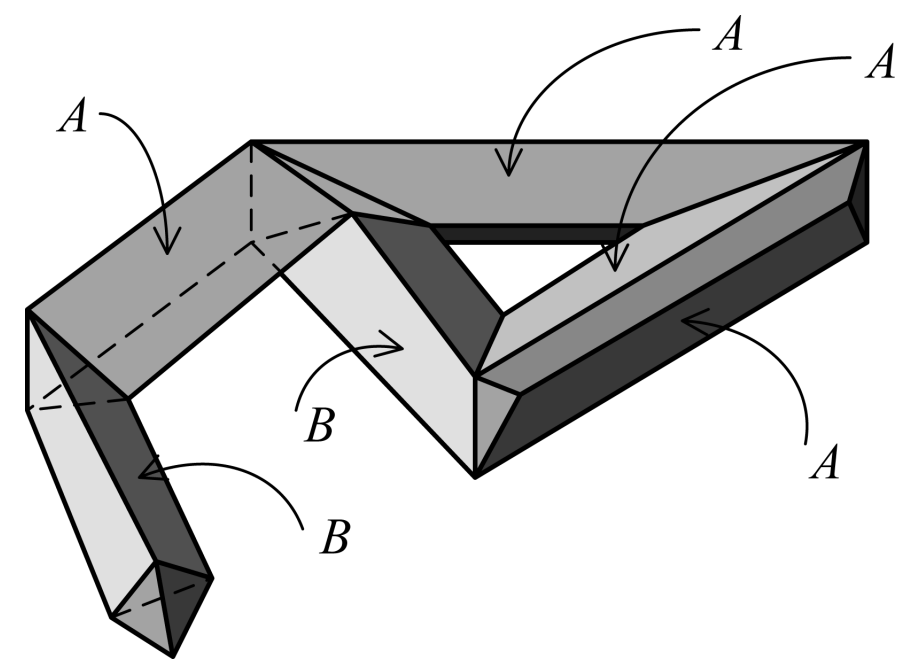

Figure 6. Piecewise convex polyhedron (1-toroid) $T_{19}$ with 19 vertices.
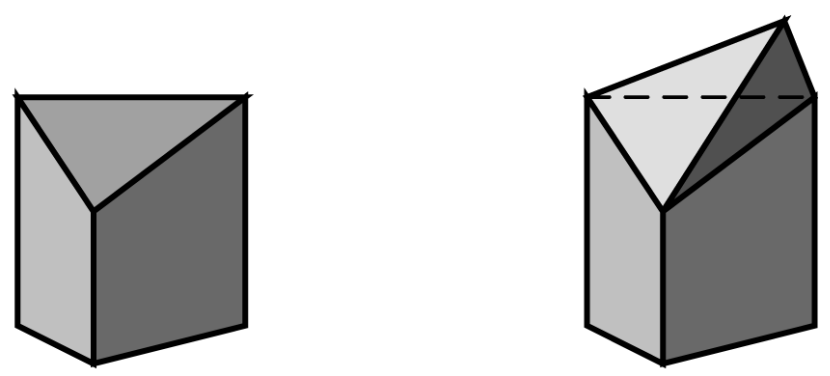

Figure 7. Elemental polyhedra of type $A$ and $B$. 
Definition 3.3. 1-toroid is cyclically piecewise convex if it is possible to divide it into cycle of convex polyhedra $P_{i}, i=1, \ldots, n$, such that $P_{i}$ and $P_{i+1}, i=1, \ldots, n-1$ and $P_{n}$ and $P_{1}$ are neighbours.

An example of cyclically piecewise convex polyhedron with $n=9$ vertices composed of three pieces of type $A$ is given in Fig. 8.

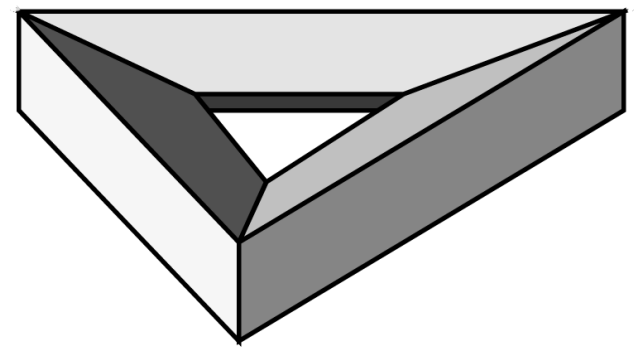

Figure 8. Cyclically piecewise convex polyhedron (1-toroid) $T_{9}$ with 9 vertices.

Remark 3.1. Division of polyhedra to convex pieces is not always unique.

For example in 1-toroid $T_{19}$, two pieces of type $A$ on the right side of the 1-toroid together build a new convex polyhedron. So, we can replace that two pieces with the new one.

Remark 3.2. Since it is always possible to 3-triangulate convex polyhedra, the same property holds for piecewise convex polyhedra, especially for piecewise convex toroids.

Remark 3.3. Each 3-triangulable polyhedron can be considered as a collection of connected tetrahedra, so it is piecewise convex.

If polyhedron $P$ is piecewise convex, let us form graph of connection of it, in such a way that nodes represent convex polyhedra $P_{i}, i=1, \ldots, m$, the pieces of $P$, while edges represent contact faces between them.

Graphs of connection for the 1-toroids $T_{9}$ and $T_{19}$ (Fig. 8 and Fig. 6) are shown in figures Fig. 9 and Fig. 10. The first graph for the 1-toroid $T_{19}$ has cyclical part and two branches, while the second one has cyclical part with only one branch, in accordance to the Remark 3.1. Since "left" branch is composed of elements $A$ and $B$ in such a way that polyhedron is not convex, making cyclical graph for $T_{19}$ is impossible.

In [22] are proved the next theorems for 1-toroids.

Theorem 3.1. For each $n \geq 7$, there exists a 1-toroid which is possible to 3-triangulate.

Theorem 3.2. If it is possible to 3-triangulate 1-toroid with $n \geq 7$ vertices, then the minimal number of tetrahedra necessary for that triangulation is $T_{\min } \geq n$. 


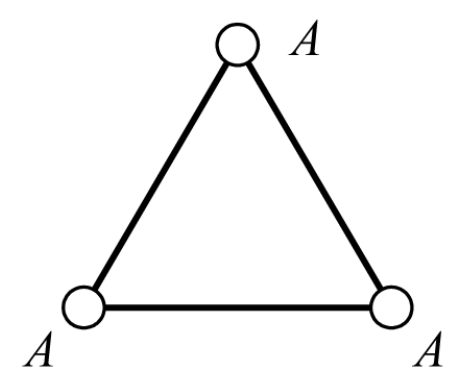

FiguRE 9. Graph of connection for the 1-toroid $T_{9}$.
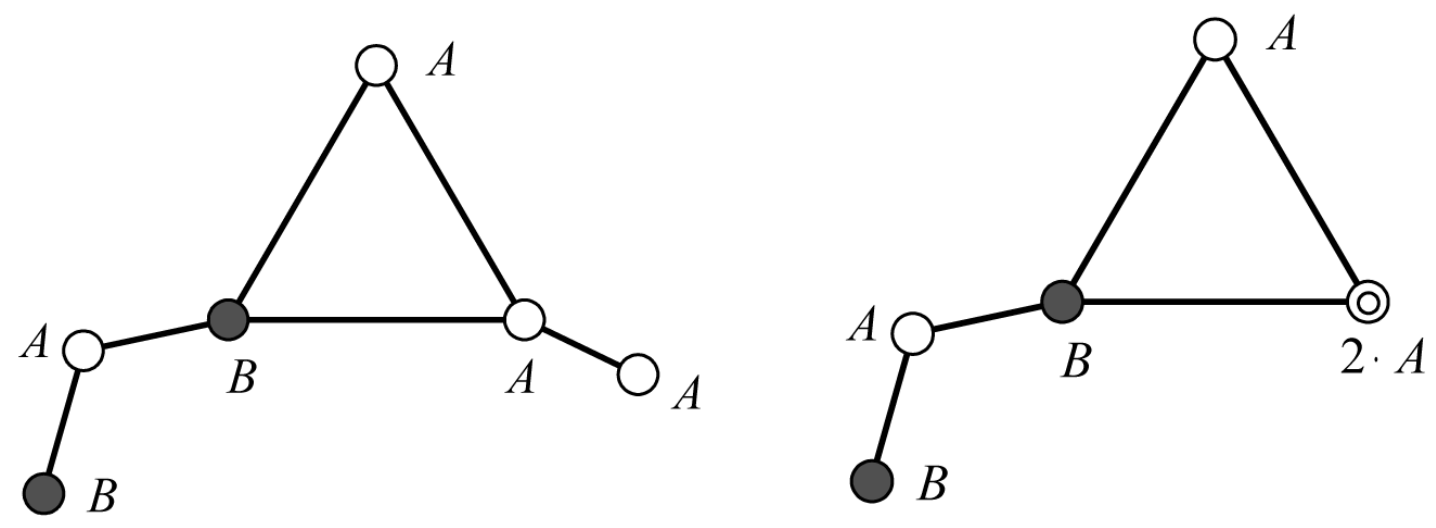

Figure 10. Two graphs of connection for the 1-toroid $T_{19}$.

Remark 3.4. In Theorem 3.2 the number $T_{\min }$ for 1 -toroid $P$ is obtained as a sum of $t_{\text {min }}^{i}, i=1, \ldots, m$, the minimal numbers of tetrahedra in 3-triangulatins of convex components $P_{i}$ of $P$. The estimation of $T_{\min }$ was made by substituting $t_{\min }^{i}$ with $n_{i}-3$ $\left(n_{i}\right.$ is number of vertices of $\left.P_{i}\right)$, since $t_{\text {min }}^{i} \geq n_{i}-3$. If we know that $t_{\text {min }}^{j}=n_{j}-3+t$ $(t>0)$, for some component $P_{j}, j=1, \ldots, m$, then it is easy to conclude that $T_{\min } \geq n+t$.

\section{3-TRIANGULATION OF 2-TOROIDS}

If 2-toroid $P$ is 3 -triangulable, then it is piecewise convex and its graph of connection $G$ has two cycles. Examples of 2-toroids are given in Fig. 11 and Fig. 12. In the first example $P_{14}$ has $n=14$ vertices and it is composed of six pieces of type $A$ or of two 1-toroids given in Fig. 8, each with $n=9$ vertices. In the second, $P_{20}$ has $n=20$ vertices and it is composed of two 1-toroids with $n=10$ vertices connected by the elemental polyhedron of type $A$. Their graphs of connection are given in Fig. 13 and Fig. 14. For $P_{14}$ there are two possible graphs, since two $A$-parts in the middle form together convex polyhedron noted with $2 \cdot A$. 


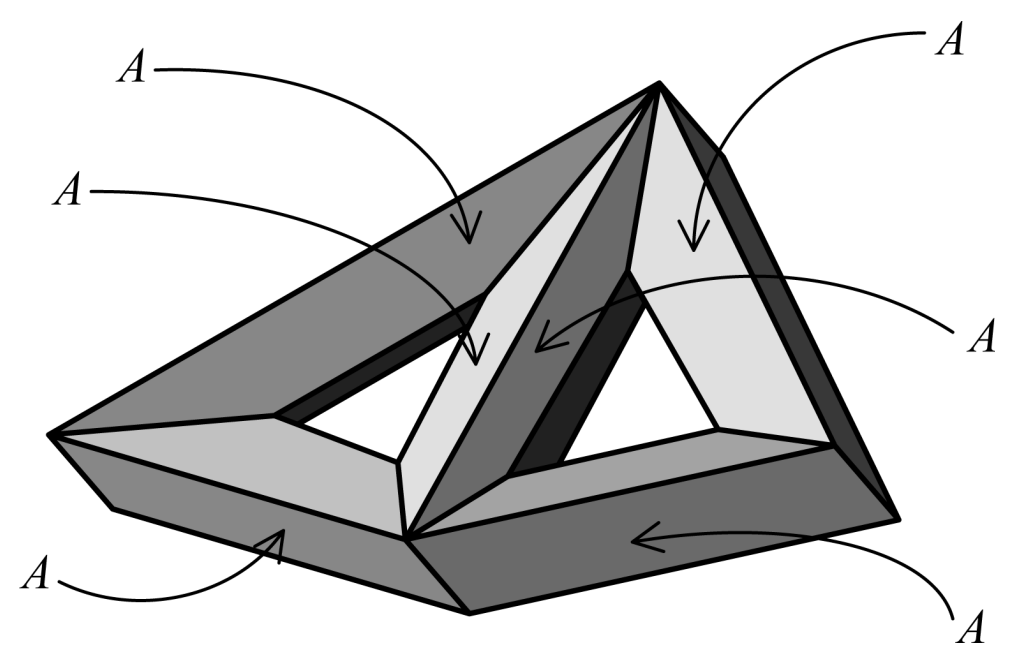

Figure 11. Piecewise convex 2-toroid $P_{14}$.

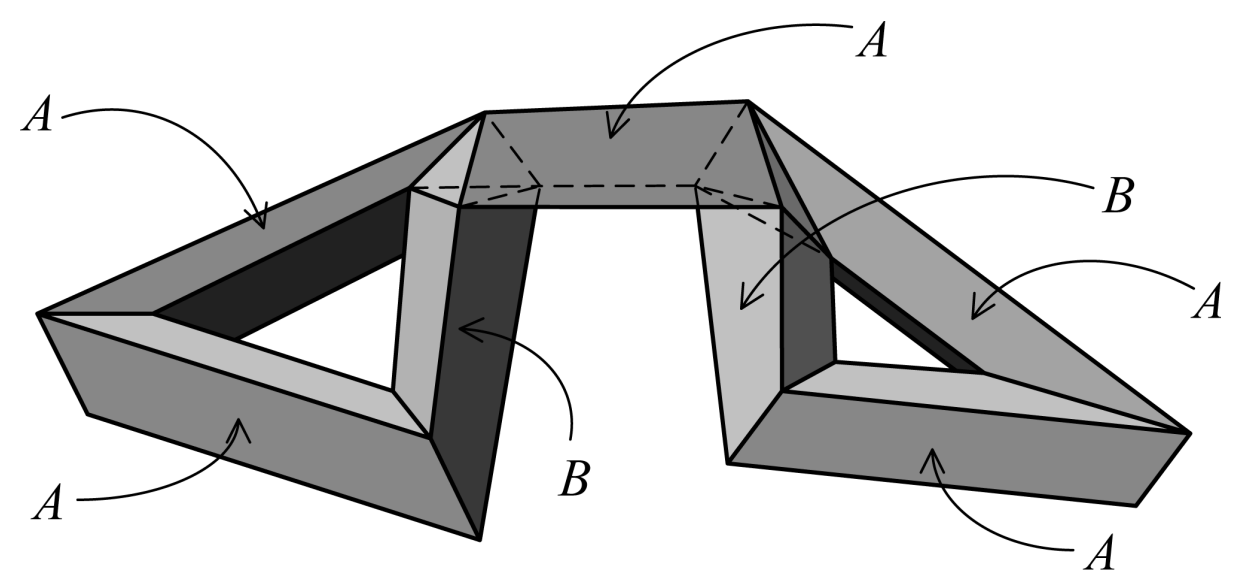

Figure 12. Piecewise convex 2-toroid $P_{20}$.
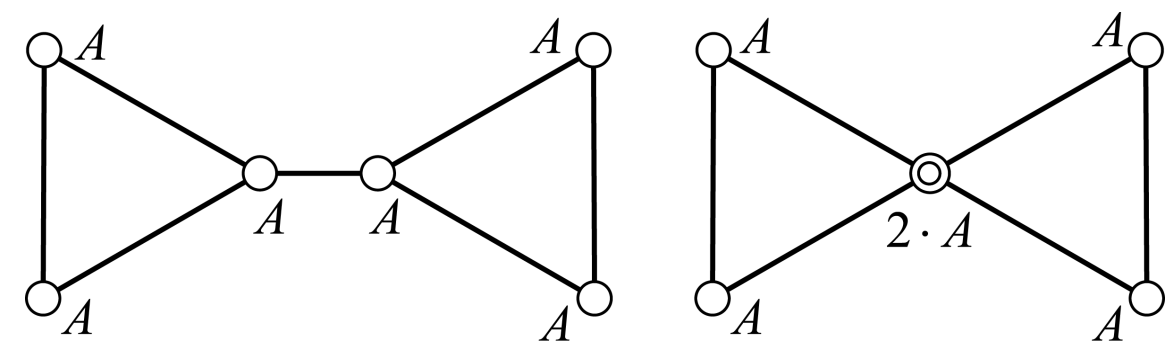

Figure 13. Two graphs of connection for the 2-toroid $P_{14}$.

Three graph examples represent three possible kinds of graphs of connection for 2 -toroids. If two cycles in the graph $G$ have a common node, it means that $P$ can be decomposed into two 1-toroids with a common convex polyhedron (as it is shown 


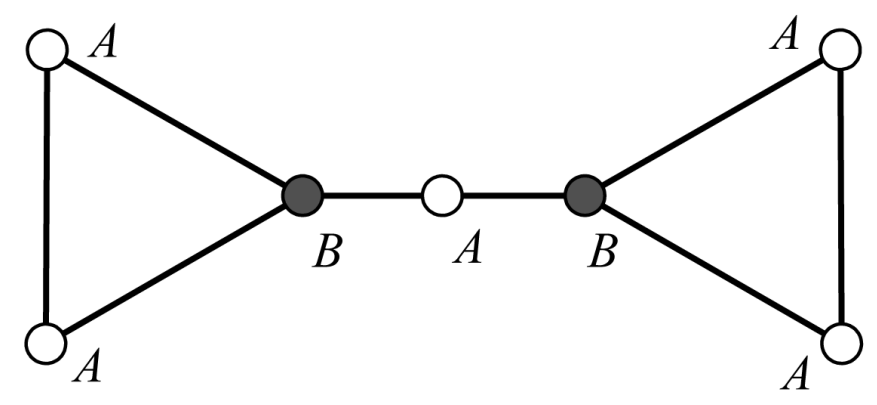

FigURE 14. Graph of connection for the 2-toroid $P_{20}$.

in the second graph in Fig. 13). If two cycles of $G$ are connected with an edge, then decomposition of the 2-toroid gives two 1-toroids with a common contact face (as it is shown in the first graph in Fig. 13). Although for $P_{14}$ it is possible to form graphs $G$ of both kinds, that is not always the situation, so we will consider that kinds of graphs separately. Finally, if two cycles of $G$ are separated with a subgraph with nodes not belonging to the cycles, then we can decompose the 2-toroid $P$ into two 1-toroids and one simple piecewise convex polyhedron, which have a common contact face with each of 1-toroids (as it is shown in Fig. 14).

It holds the next theorem.

Theorem 4.1. For each $n \geq 10$ there exists a 2-toroid which is possible to 3triangulate.

Proof. (Description of piecewise 2-toroids with $n \geq 10$ vertices).

1. Considering graphs of connection, we conclude that the 3 -triangulable 2-toroid $P$ with the smallest number of vertices has to be composed of two Császár polyhedra with the common convex polyhedron. Since Császár polyhedron has no diagonals, the same property has to be fulfilled to its part, which would be used as the common convex polyhedron. But the only simple convex polyhedron without diagonals is tetrahedron [16]. It means that such a 2-toroid, let us call it double-Császár, has $n=2 \cdot 7-4=10$ vertices, and that it is triangulable with $t=2 \cdot 7-1=13$ tetrahedra.

"Gluing" tetrahedra, one by one, to double-Császár polyhedron, we can form a 2-toroid $P$ for each $n>10$. Graphs of such 2-toroids $P$ would have two 1-cycles connected by the common node and the additional tree/trees connected to some of the other nodes.

2. Other possibility to form piecewise 2 -toroids $P$ with $n \geq 14$ vertices is to "glue" two of 1-toroids with $n \geq 9$ vertices from the series $\mathbf{S}$ described below, in such a way to get a common quadrangular face.

We can obtain 1-toroids from $\mathbf{S}$ with $n=3 k, k \geq 3$ vertices by cyclically connecting $k$ polyhedra of type $A$. Starting 1-toroid $T_{9}$ from this series is given in Fig. 8. If 
instead of one or two polyhedra $A$ we put in the cycle polyhedron/polyhedra $B$, then 1-toroid from $\mathbf{S}$ would have $n=3 k+1$ or $n=3 k+2$ vertices.

We note with $n_{1}$ and $n_{2}\left(n_{1}, n_{2} \geq 9\right)$ numbers of vertices of 1 -toroids $T_{1}$ and $T_{2}$ from $\mathrm{S}$ used to form 2-toroid $P$. Then, number of vertices of $P$ would be $n=n_{1}+n_{2}-4$, since $T_{1}$ and $T_{2}$ have four common vertices. 2-toroid made in this way with the smallest number of vertices is $P_{14}$, shown in Fig. 11.

In [22] was proved that minimal triangulation of 1-toroid from series $\mathbf{S}$ with $n$ vertices have $n$ tetrahedra. It follows that for described 2-toroid $P, T_{\min }=n_{1}+n_{2}=$ $n+4$.

3. If each of two 1-toroids, $T_{1}$ and $T_{2}$, from series $\mathbf{S}$ would have two triangular faces forming space quadrangle, instead of one face with a flat quadrangle, than we can form 2-toroid $P$ by gluing $T_{1}$ and $T_{2}$ through their common triangular face, a half of previous quadrangular. Such $P$ would have $n=n_{1}+n_{2}-3$ vertices and $T_{\min }=n_{1}+n_{2}=n+3$. The smallest number of vertices of $P$ obtained in this way can be 15 .

For the minimal number of tetrahedra in triangulation, the next theorem holds.

Theorem 4.2. If it is possible to 3-triangulate 2-toroid with $n \geq 10$ vertices then, the minimal number of tetrahedra necessary for triangulation is $T_{\min } \geq n+3$.

Proof. Each of the three mentioned situations of the graph $G$ will be considered separately.

1. If the graph $G$ of the 2-toroid $P$ is with a common node for two cycles, then let us note with $n_{1}, n_{2}$ the numbers of vertices of two 1-toroids and with $n_{3}$ of common convex polyhedron. The numbers of tetrahedra in the minimal triangulation of 1-toroids will be noted with $t_{1}, t_{2}$, and with $t_{3}$ of their common convex polyhedron.

If $t_{3}=n_{3}-3$, then by theorem $3.2, t_{1} \geq n_{1}$ and $t_{2} \geq n_{2}$. When $t_{3}=n_{3}-3+t$, $t>0$, since the convex polyhedron is a part of both 1-toroids, by the theorem 3.2 and remark 3.4, it follows that $t_{1} \geq n_{1}+t$ and $t_{2} \geq n_{2}+t$.

We may assume that $t_{1} \geq n_{1}+t, t_{2} \geq n_{2}+t$ with $t \geq 0$. Number of vertices of the 2-toroid $P$ is

$$
n=n_{1}+n_{2}-n_{3}
$$

while the number of tetrahedra in the minimal triangulation is

$$
\begin{aligned}
T_{\text {min }} & =t_{1}+t_{2}-t_{3} \geq \\
& \geq n_{1}+t+n_{2}+t-\left(n_{3}-3+t\right) \\
& =n_{1}+n_{2}-n_{3}+3+t \\
& =n+3+t, \quad t \geq 0 .
\end{aligned}
$$

So, it holds $T_{\min } \geq n+3$.

2. When the cycles of $G$ are connected by an edge, then two constitutive 1-toroids $T_{1}$ and $T_{2}$ in composition have $n_{1}$ and $n_{2}$, and the contact face has $m \geq 3$ vertices. If numbers of tetrahedra in the minimal triangulations of 1-toroids are noted by $t_{1}$ 
and $t_{2}$, then by theorem $3.2, t_{1} \geq n_{1}$ and $t_{2} \geq n_{2}$. In the minimal triangulation of 2-toroid, $P$, the number of tetrahedra is $T_{\min } \leq t_{1}+t_{2}$. If $m=3$ or $m=4$, then

$$
T_{\text {min }}=t_{1}+t_{2} \geq n_{1}+n_{2}=n+m \geq n+3
$$

always holds. But, when $m>4$, it may happen that $T_{\min }<t_{1}+t_{2}$. We have such a situation if it is possible to form a convex (or piecewise convex) polyhedron $S$, which is 3-triangulable with smaller number of tetrahedra around the contact face, then in its 3 -triangulation is induced by those used to separate 1-toroids $T_{1}$ and $T_{2}$. Such a polyhedron $S$, can be bipyramid with 5 or more vertices in the basis, shown in section 2.4. Here, the basis of bipyramid would be used as the contact face.

Using new, alternative 3-triangulation of $S$, we can induce different decompositions of $P$ with graph $G^{\prime}$, having a common node of cycles. It means, as in the previous case, that in both situations $T_{\min } \geq n+3$.

3. In the third case of the graph $G$, the number of vertices of $P$ is $n=n_{1}+n_{2}+n_{3}-$ $m_{1}-m_{2}$, where $n_{1}, n_{2}$ are numbers of vertices for the 1-toroids, $n_{3}$ for the simple polyhedron between them, while $m_{1} \geq 3, m_{2} \geq 3$ are numbers of vertices of the contact faces of the 1-toroids and the simple polyhedron.

Since, hold $t_{1} \geq n_{1}, t_{2} \geq n_{2}$ for the numbers of tetrahedra in the minimal triangulations of the 1-toroids, and $t_{3} \geq n_{3}-3$ for the simple polyhedron, then

$$
T_{\text {min }}=t_{1}+t_{2}+t_{3} \geq n_{1}+n_{2}+n_{3}-3 \geq n+3 .
$$

Here is again assumed that simplifications like those in case 2. are not possible. Else, when $T_{\min }<t_{1}+t_{2}+t_{3}$ alternative "simpler" decomposition of $P$ is possibly, with simplified graph $G^{\prime}$, possible belonging to case 1 . or 2 .

So, again $T_{\min } \geq n+3$ holds.

4. For 1-toroids in [22] was proved that existence of additional branches in its graph of connection would not change estimated value $T_{\min }$. Here, we may assume that if any additional branch appears in graph $G$ of $P$, it belongs either to 1-toroid $T_{1}$ or to $T_{2}$. It means that even than, for $P$ holds $T_{\min } \geq n+3$.

Remark 4.1. In considering different decompositions of polyhedron $P$ into convex pieces, we may concentrate only to these by tetrahedra in the minimal triangulation. Then collections of tetrahedra corresponding to nodes in the cycles of such graph $G$ form two 1-toroids $P_{1}, P_{2}$, which are cyclically piecewise convex, while other tetrahedra form simple piecewise convex polyhedra $P_{i}, i \geq 3$, one for each branch of $G$. In such a decomposition, $T_{\min }$ is equal to sum of $t_{\text {min }}^{i}$, the numbers of tetrahedra in the minimal triangulations of pieces $P_{i}, i \geq 1$.

Theorem 4.3. For each $n \geq 10$ there exist 2-toroid $P$ with $n$ vertices and $T_{\min }=n+3$.

Proof. As it is mentioned in part 3. of proof of theorem 4.1, for all there constructed 2 -toroids with $n \geq 15$ vertices, it holds $T_{\min }=n+3$. In part 1 . of the same proof the double-Császár 2 -toroid with $n=10$ vertices and $T_{\min }=13$ is introduced. 
For some of others 2-toroids described in 1. also hold $T_{\min }=n+3$. The mentioned property holds whenever added branches in graph of connection are joined to (simple) convex polyhedra $P_{i}$ with $t_{\text {min }}^{i}=n_{i}-3$, if contact faces are triangular. So, we can introduce 2-toroids with desired property for $n=11,12,13,14$ if we glue one or two tetrahedra or bipyramids with a triangular basis to the double-Császár 2-toroid. Note that gluing each tetrahedron brings one new vertex, while gluing a bipyramid with a triangular basis brings two new vertices. More precise, we can form 2-toroids with the mentioned property by gluing: for $n=11$ one tetrahedron, for $n=12$ two tetrahedra or one bipyramid, for $n=13$ one tetrahedron and one bipyramid and for $n=14$ two bipyramids.

We can also obtain 2-toroids with property $T_{\min }=n+3$ if instead of the doubleCsászár 2-toroid we use other basic 2-toroid. For example, we can glue two Császár 1-toroids. Then common face is triangular, so the new 2-toroid would have 11 vertices. If elemental polyhedron A would be inserted between two Császár 1-toroids (glued to 1-toroids by its triangular faces), then the number of vertices would be 14 . With inserted elemental polyhedron $\mathrm{B}$, the number of vertices would be 15 . Further gluing simple convex polyhedra $P_{i}$ with $t_{\min }^{i}=n_{i}-3$ to the basic 2 -toroid with $11(14,15)$ vertices will give new 2 -toroids with property $T_{\min }=n+3$ and $n \geq 12(n \geq 15$, $n \geq 16)$ vertices.

\section{Summary}

The concept of the piecewise convex polyhedron is useful in considering 3-triangulation of non-convex polyhedra, especially of toroids. We can do 3-triangulation by using graph of connection of these polyhedra. In this paper, we discussed the problems of existence of 3-triangulable 2-toroids, and of the minimal number of tetrahedra necessary for their 3 -triangulation. In the similar way, it would be possible to investigate the same problems for polyhedra topologically equivalent to sphere with $p$ handles but with more possible cases of graphs of connection. For $p$-toroids it would be difficult to establish the minimal number of vertices necessary to form such a polyhedron. Since this investigation is more complicated, it would be left out for some future work.

\section{REFERENCES}

[1] D. Avis and H. ElGindy, Triangulating point sets in space, Discrete Comput. Geom. 2 (1987), 99-111.

[2] J. Bokowski and A. Eggart, All realizations of Möbius torus with 7 vertices, Structural Topology 17 (1991), 59-78.

[3] J. Bokowski, On tori of Császár and Szilassi and more, Visual Mathematics (2005), http://www.mi.sanu.ac.rs/vismath/visbook/bokowsky.

[4] B. Chazelle, Triangulating a simple polygon in linear time, Discrete Comput. Geom. 6(5) (1991), $485-524$

[5] A. Császár, A polyhedron without diagonals, Acta Sci. Math. Universitatis Szegediensis 13 (1949), 140-142. 
[6] F. Y. L. Chin, S. P. Y. Fung and C. A. Wang, Approximation for minimum triangulations of simplicial convex 3-polytopes, Discrete Comput. Geom. 26(4) (2001), 499-511.

[7] M. Develin, Maximal triangulations of a regular prism, J. Comb. Theory, Ser. A 106(1) (2004), $159-164$.

[8] H. Edelsbrunner, Algorithms in Combinatorial Geometry, Springer-Verlag, Heidelberg (1987).

[9] H. Edelsbrunner, F. P. Preparata and D. B. West, Tetrahedrizing point sets in three dimensions, J. Symbolic Computation 10 (1990), 335-347.

[10] C. W. Lee, Subdivisions and triangulations of polytopes, Handbook of Discrete and Computational Geometry (J.E. Goodman and J. O'Rourke eds.), CRC Press, New York, (1997), pp. 271-290.

[11] J. Rupper and R. Seidel, On the difficulty of triangulating three-dimensional nonconvex polyhedra, Discrete Comput. Geom. 7 (1992), 227-253.

[12] E. Schönhardt, Über die Zerlegung von Dreieckspolyedern in Tetraeder, Math. Ann. 98 (1928), 309-312.

[13] R. Seidel, A simple and fast incremental randomized algorithm for computing trapezoidal decompositions and for triangulating polygons, Computational Geometry 1(1) (1991), 51-64.

[14] D. D. Sleator, R. E. Tarjan and W. P. Thurston, Rotation distance, triangulations, and hyperbolic geometry, J. of the Am. Math. Soc. 1(3) (1988), 647-681.

[15] S. Szabó, Polyhedra without diagonals, Period. Math. Hung. 15 (1984), 41-49.

[16] S. Szabó, Polyhedra without diagonals II, Period. Math. Hung. 58(2) (2009), 181-187.

[17] L. Szilassi, Regular toroids, Structural Topology 13 (1986), 69-80.

[18] L. Szilassi, On some regular toroids, Visual Mathematics (2005), http://www.mi.sanu.ac.rs/vismath/visbook/szilassi.

[19] L. Szilassi, The Császár polyhedron subdivided into tetrahedra, Wolfram Demonstrations Project (2012), http://demonstrations . wolfram. com/TheCsaszarPolyhedronSubdividedIntoTetrahedra.

[20] M. Stojanović, Algorithms for triangulating polyhedra with a small number of tetrahedra, Mat. Vesnik 57 (2005), 1-9.

[21] M. Stojanović, Triangulations of some cases of polyhedra with a small number of tetrahedra. Kragujevac J. Math. 31 (2008), 85-93.

[22] M. Stojanović, On 3-triangulation of toroids, Filomat 29(10) (2015), 2393-2401.

[23] M. Stojanović and M. Vučković, Algorithms for investigating optimality of use cone triangulation for a given polyhedron, Kragujevac J. Math. 30 (2007), 327-342.

[24] M. Stojanović and M. Vučković, Convex polyhedra with triangular faces and cone triangulation, YUJOR 21(1) (2011), 79-92.

${ }^{1}$ Faculty of Organizational Sciences

Jove IlićA 154,

11040 Belgrade, Serbia

E-mail address: milicas@fon.bg.ac.rs 